\title{
Prediction of identity by descent probabilities from marker-haplotypes
}

\author{
Theo H.E. Meuwissen ${ }^{\mathrm{a}, *}$, Mike E. Goddard ${ }^{\mathrm{b}}$ \\ ${ }^{a}$ Research Institute of Animal Science and Health, \\ Box 65, 8200 AB Lelystad, The Netherlands \\ b Institute of Land and Food Resources, University of Melbourne, Parkville \\ Victorian Institute of Animal Science, Attwood, Victoria, Australia
}

(Received 13 February 2001; accepted 11 June 2001)

\begin{abstract}
The prediction of identity by descent (IBD) probabilities is essential for all methods that map quantitative trait loci (QTL). The IBD probabilities may be predicted from marker genotypes and/or pedigree information. Here, a method is presented that predicts IBD probabilities at a given chromosomal location given data on a haplotype of markers spanning that position. The method is based on a simplification of the coalescence process, and assumes that the number of generations since the base population and effective population size is known, although effective size may be estimated from the data. The probability that two gametes are IBD at a particular locus increases as the number of markers surrounding the locus with identical alleles increases. This effect is more pronounced when effective population size is high. Hence as effective population size increases, the IBD probabilities become more sensitive to the marker data which should favour finer scale mapping of the QTL. The IBD probability prediction method was developed for the situation where the pedigree of the animals was unknown (i.e. all information came from the marker genotypes), and the situation where, say $T$, generations of unknown pedigree are followed by some generations where pedigree and marker genotypes are known.
\end{abstract}

identity by descent / haplotype analysis / coalescence process / linkage disequilibrium / QTL mapping

\section{INTRODUCTION}

Often, a gene for a discrete or quantitative trait is mapped relative to genetic markers but not identified [15]. The mapping and subsequent investigation of the mapped gene depends on the ability to predict whether two animals or gametes are carrying the same allele at this gene because they are identical by descent (IBD; e.g. [9]). For instance, the classical gene mapping experiment can be described as determining whether animals carrying alleles which are

\footnotetext{
* Correspondence and reprints

E-mail: t.h.e.meuwissen@id.dlo.nl
} 
identical by descent (based on markers) are more similar than random animals for the trait of interest. If the markers are in linkage equilibrium with the gene, then IBD can only be traced with the use of pedigree information as well as marker genotypes. For example, in a daughter design for QTL mapping, genetic markers are used to trace which daughters of a sire carry a chromosome region that are IBD [24]. However, if the markers and the gene are in Linkage Disequilibrium (LD), then chromosomes carrying the same markers are likely to be carrying the same alleles at the gene as well, which is for instance utilised by the Transmission Disequilibrium Test $[17,19]$. In this situation the IBD status of the chromosome regions can be predicted even without pedigree information. In practice, some pedigree data is likely to be known but it will be desirable to also make use of linkage disequilibria which result from more distant relationships than those in the recorded pedigree, and here emphasis will be on this LD information. However the IBD probabilities are calculated, they are the fundamental data for mapping the gene more finely or estimating its effect on traits of interest, or using the markers for marker assisted selection or genetic counselling. This becomes most apparent in the variance component methods for QTL mapping $(e . g$. $[9,14])$, where the matrix of IBD probabilities given the marker information is used as a correlation matrix between the random effects of the multi-allelic QTL (e.g. $[9,14])$. However, for full maximum likelihood QTL mapping, the pairwise IBD probabilities between haplotypes do not contain all necessary information.

Information based on LD is more useful if several closely linked markers defining a haplotype are used to mark the chromosome region [21]. Consider a gene, denoted $A$, that is known to map within the region spanned by a set of five markers. Two gametes that share the same marker haplotype (say 1111 1) are more likely than random gametes to share alleles at $A$ that are IBD, but how much more likely? If these two gametes descend from a common great grandfather, how does this affect the probability that they have $A$ alleles that are IBD? The purpose of this paper is to propose a method for calculating the probability that gametes are IBD at a chromosome location based on marker haplotypes from the same chromosomal region. In a previous paper [14], we used simulation to estimate this probability and assumed that no pedigree information was available. Here we present an analytical method and include the use of pedigree data if it is available.

\section{METHODS}

The derivation assumes a random mating population of effective size $N_{\mathrm{e}}$ that descended from a base generation $T$ generations ago. The alleles at the marker loci were approximately in linkage equilibrium in the base population. We considered two haplotypes from this population, observed their marker alleles, 
and calculated the probability that the two haplotypes are IBD at some locus of interest, which was denoted by locus $A$. The haplotypes were assumed randomly sampled, and may or may not come from the same individual. We have considered the situation where the haplotype consisted of one marker locus and locus $A$ and ignored the pedigree information, and later extended this to more marker loci and included pedigree information. When pedigree information was available there were still founder individuals at the top of the pedigree who had no known ancestors. LD was used to estimate the IBD probabilities among the QTL alleles carried by these founders.

\subsection{IBD probability at locus $A$ given one linked marker}

The method calculates IBD probabilities at locus $A$ back to an arbitrary base population $T$ generations ago. Let $S$ be an indicator of the Alike In State (AIS) situation of the marker alleles, i.e. $S=1(S=0)$ indicates the alleles are AIS (nonAIS). Note that if $S=1$, the marker locus may still be IBD or nonIBD. Now, the probability that the alleles at locus $A$ are IBD given the marker data is:

$$
P(\operatorname{IBD} \mid \text { marker })=P(\operatorname{IBD} \mid S)=\frac{P(A=\operatorname{IBD} \& S)}{P(A=\operatorname{IBD} \& S)+P(A=\operatorname{nonIBD} \& S)}
$$

i.e., we have to calculate terms like $P(S \& A=$ non IBD).

Next we defined a character string $\phi$ of three characters which summarises the IBD status of the region which was spanned by the loci. Table I demonstrates the use of $\phi$. More precisely, $\phi(1)$ and $\phi(3)$ are 1 or 0 indicating whether locus $A$, and the marker locus, respectively, are IBD or not. The in between character $\phi(2)=$ "_" indicates that the region in between the two loci is IBD due to the same common ancestor as the loci, i.e. the region in between the markers was inherited as a whole from the same common ancestor without a recombination that splits the region. $\phi(2)=$ " $\mathrm{x}$ " indicates that there has been a recombination and, if the two loci are IBD, they are probably IBD due to different common ancestors. It is important to distinguish $\phi=$ "1_1" from $\phi=$ " $1 \mathrm{x} 1$ ", because the probability that the region was inherited as a whole from the same ancestor differs from the probability that both loci are IBD due to different common ancestors. If either $\phi(1)$ or $\phi(3)$ or both are 0 , we must have $\phi(2)=$ " $\mathrm{x}$ " because at least (a small) part of the region is not IBD. Note that if a recombination occurs in an individual that is inbred for the entire region, $\phi=$ "1x1" and not "1_1", although $\phi=$ "1_1" would yield the same genotype in this case (this convention simplifies the calculation of $P(\phi=$ "1_1"), which involves the calculation of the probability of no recombination since the most recent common ancestor, while it would otherwise involve the calculation of no recombination in a non-inbred individual, which is more complicated). 
Table I. Illustration of the similarity vector $S$, the IBD status indicator $\phi$, and the conditional probability of $S$ given $\phi(P(S \mid \phi))$ in the case of two loci.

The first locus refers to locus $A$ and the second to the marker locus. Note that if $S$ indicates that the marker alleles are unequal, $\phi$ has to indicate a nonIBD marker locus, but if the marker alleles are equal the marker locus may be IBD or nonIBD.

\begin{tabular}{llcc}
\hline $\begin{array}{l}\text { Marker Alike } \\
\text { in State: }\end{array}$ & Locus $A$ & $\begin{array}{c}\text { Possible } \\
\phi^{(\mathrm{a})}\end{array}$ & $P(S \mid \phi)^{(\mathrm{b})}$ \\
\hline$S=0$ & nonIBD & $0 \times 0$ & $1-a_{i}$ \\
& IBD & $1 \times 0$ & $1-a_{i}$ \\
$S=1$ & & & \\
& nonIBD & $0 \times 1$ & 1 \\
& & $0 \times 0$ & $a_{i}$ \\
& IBD & $1 \_1$ & 1 \\
& & $1 \times 1$ & 1 \\
& & $1 \times 0$ & $a_{i}$ \\
\hline
\end{tabular}

(a) $\phi=$ "0x0" denotes that both loci are nonIBD; $\phi=$ " $1 \mathrm{x} 0$ " denotes that the first locus is IBD and the second is nonIBD; $\phi=$ "1_1" denotes that both loci and the in between region are IBD and as a whole inherited from one common ancestor; $\phi=$ "1x1" denotes that both loci are IBD but there has been a recombination in the in between region, such that the loci are (most likely) IBD due to different common ancestors.

(b) $a_{i}=$ probability of the marker locus $i$ being alike in state. Hence, if $\phi$ indicates an nonIBD marker locus, the marker alleles may still be equal $(S=1)$ with probability $a_{i}$, and thus unequal $(S=0)$ with probability $1-a_{i}$.

Now $P(S \& A=$ IBD) can be obtained by summing over all possible IBD statuses, $\phi$, with locus $A=\mathrm{IBD}$ :

$$
P(S \& A=\mathrm{IBD})=\sum_{\phi \mid \phi(1)=1} P(S \mid \phi) \times P(\phi),
$$

similarly:

$$
P(S \& A=\text { nonIBD })=\sum_{\phi \mid \phi(1)=0} P(S \mid \phi) \times P(\phi),
$$

where $\sum_{\phi \mid \phi(1)=1}\left(\sum_{\phi \mid \phi(1)=0}\right)$ denotes summation over all possible $\phi$ vectors where locus $A$ is (non)IBD; $P(S \mid \phi)=$ the probability of AIS markers denoted by $S$ given the IBD statuses denoted by $\phi$ (see Tab. I).

The probabilities of the marker alleles being identical given the IBD status of the marker locus are shown in Table I, except for the case where the marker alleles are IBD but unequal which is impossible. As shown in Table I, $P(S \mid \phi)$ can involve the probability that the alleles at locus $i$ are alike in state, which is denoted by $a_{i}$. For nonIBD marker alleles, the probability of being alike in state equals the homozygosity at locus $i$ in the base generation, $a_{i}$. 
Equations (2) also involve the calculation of $P(\phi)$. We first consider $\phi=\left[1 \_1\right]$, i.e., the chromosome segment between and including both loci is inherited from a common ancestor. $P\left(\phi=\left[1 \_1\right]\right)$ is calculated by an argument analogous to that used in coalescence theory $[10,11]$ in which we trace back the (unknown) pedigree of both haplotypes until a common ancestor occurs, say, $t$ generations ago. The probability of having no common ancestor for $t-1$ generations is $\left(1-1 /\left(2 N_{\mathrm{e}}\right)\right)^{t-1}$ and one in generation $t$ is $1 /\left(2 N_{\mathrm{e}}\right)$, where $N_{\mathrm{e}}$ is the effective population size. Furthermore, we require that there was no recombination within this chromosome segment in both paths that descend from the common ancestor for $t$ generations, which has a probability of $[\exp (-c)]^{2 t}$, where $\exp (-c)$ is the probability of no recombination during one meiosis assuming a Poisson distribution of recombinations, and $c$ is the distance between the loci (in Morgans). Combining these probabilities yields the probability of a common ancestor $t$ generations ago and no recombination since over a region of $c$ Morgan:

$$
\frac{1}{2 N_{\mathrm{e}}}\left(1-\frac{1}{2 N_{\mathrm{e}}}\right)^{t-1}(\exp [-c])^{2 t} \approx \frac{1}{2 N_{\mathrm{e}}} \exp \left[-\frac{t-1}{2 N_{\mathrm{e}}}-2 c t\right] .
$$

The common ancestors may have occurred in any of the generations between the base population and the present population, i.e. $t=1,2, \ldots, T$, where $T$ is the number of generations since the unrelated base population. Hence, the probability of having an IBD region of size $c$ is:

$$
\begin{aligned}
\mathrm{f}(c) & =\frac{1}{2 N_{\mathrm{e}}} \exp [-2 c] \sum_{t=1}^{T} \exp \left[-(t-1)\left(\frac{1}{2 N_{\mathrm{e}}}+2 c\right)\right] \\
& =\frac{\exp [-2 c]}{2 N_{\mathrm{e}}} \times \frac{1-\exp \left[-T\left(2 c+\frac{1}{2 N_{\mathrm{e}}}\right)\right]}{1-\exp \left[-\left(2 c+\frac{1}{2 N_{\mathrm{e}}}\right)\right]}
\end{aligned}
$$

where $\mathrm{f}(c)=$ coefficient of kinship for a region of size $c$. Note that the IBD region may extend beyond the chromosome segment of size $c$, and that $\mathrm{f}(0) \approx 1-\exp \left(-T /\left(2 N_{\mathrm{e}}\right)\right)$, i.e. the coefficient of kinship of a region of size 0 , i.e. at a locus, equals approximately the inbreeding coefficient in generation $T$. Equation (3) is a simplification of the coalescence process in that 1) generations are assumed discrete instead of continuous; and 2) it refers to a base population $T$ generations ago to avoid that all alleles are IBD, while the coalescence process simulates mutation to achieve this.

The probability that the entire region between locus $A$ and the marker is IBD is thus:

$$
P\left(\phi=\left[1 \_1\right]\right)=\mathrm{f}(c) .
$$


Next we will consider the case where $\phi=[1 \times 1]$, i.e., the marker locus and locus $A$ are IBD but the region in between them has recombined. Hence, at locus $A$ we have an IBD region that is bounded on the right side. The probability of an IBD region of size $c$ with one (or more) recombination on the right (or left) side in a region of size $c_{1}$, will be denoted by $\mathrm{f}_{\mathrm{r}}\left(c, c_{1}\right)$. The probability $\mathrm{f}_{\mathrm{r}}\left(c, c_{1}\right)$ is easily obtained from the equation:

$$
\begin{aligned}
\mathrm{f}(c)= & P(\text { IBD \& No recomb. over region of size } c) \\
= & P(\text { IBD \& No recomb. over region of size } c \\
& \left.\& \text { No recomb. in next region of size } c_{1}\right) \\
& +P(\text { IBD \& No recomb. over region of size } c \\
& \left.\& \text { recomb. in next region of size } c_{1}\right) \\
= & \mathrm{f}\left(c+c_{1}\right)+\mathrm{f}_{\mathrm{r}}\left(c, c_{1}\right) .
\end{aligned}
$$

It follows that:

$$
\mathrm{f}_{\mathrm{r}}\left(c, c_{1}\right)=\mathrm{f}(c)-\mathrm{f}\left(c+c_{1}\right),
$$

where $\mathrm{f}(c)$ and $\mathrm{f}\left(c+c_{1}\right)$ are from equation (3). Similarly, the probability of having an IBD region of size $c$, that is bounded on both sides in regions of size $c_{1}$ (to the left) and $c_{2}$ (to the right) is:

$$
\mathrm{f}_{\mathrm{dr}}\left(c, c_{1}, c_{2}\right)=\mathrm{f}_{\mathrm{r}}\left(c, c_{1}\right)-\mathrm{f}_{\mathrm{r}}\left(c+c_{2}, c_{1}\right) .
$$

If $\phi=$ [1x1], we first have an IBD region of size 0 around locus $A$ which ends in a region of size $c$. The latter has a probability of $\mathrm{f}_{\mathrm{r}}(0, c)$. After this region of size $c$, which contains a recombination, the marker locus is IBD again. We will assume that the recombination makes the probability of an IBD marker locus approximately independent of the IBD status of locus $A$, i.e. the probability of an IBD marker locus is $f(0)$, which is the coefficient of coancestry at a single locus (and equals approximately the coefficient of inbreeding). This assumption of an independent locus after a recombination will be examined in detail in Section 4. DISCUSSION. It follows that the probability of $\phi=[1 \mathrm{x}$ ?] is $\mathrm{f}_{\mathrm{r}}(0, c)$ and $P(\phi=[1 \mathrm{x} 1] \mid \phi=[1 \mathrm{x}$ ? $]) \approx \mathrm{f}(0)$, where the "?"-sign denotes an undetermined IBD status. Combining these probabilities yields:

$$
\begin{aligned}
P(\phi=[1 \mathrm{x} 1]) & =P(\phi=[1 \mathrm{x} ?]) P(\phi=[1 \mathrm{x} 1] \mid \phi=[1 \mathrm{x} ?]) \\
& =\mathrm{f}_{\mathrm{r}}(0, c) \mathrm{f}(0) .
\end{aligned}
$$

Next consider $\phi=[1 \mathrm{x} 0]$ : the probability that the first locus is IBD followed by a recombination is as before $\mathrm{f}_{\mathrm{r}}(0, c)$. The second locus is again independent due to the recombination between the loci and is nonIBD with probability $1-\mathrm{f}(0)$. Combining these probabilities yields:

$$
P(\phi=[1 \mathrm{x} 0])=\mathrm{f}_{\mathrm{r}}(0, c)(1-\mathrm{f}(0)) .
$$


Table II. Calculation of IBD probability between two gametes at locus $A$ given that a linked marker has identical alleles.

The effective size and time since the base population are both 100 , the distance between both loci is $0.01 \mathrm{M}$, and initial homozygosity of the marker was 0.5 . Equation numbers are in parentheses ().

\begin{tabular}{lccc}
\hline & & $A$ is IBD: & $A$ is nonIBD: \\
IBD-status $(\phi){ }^{(\text {a) }}$ & $P(\phi)$ & $P(S \mid \phi)$ & $P(S \mid \phi)$ \\
\hline $1 \_1$ & $0.1822(4)$ & 1 & - \\
$1 \times 1$ & $0.0837(5)$ & 1 & - \\
$1 \times 0$ & $0.1285(6)$ & 0.5 & 1 \\
$0 \times 1$ & $0.1285(6)$ & - & 0.5 \\
$0 \times 0$ & $0.4778(8)$ & - & $0.3674(2)$ \\
\hline$\sum P(\phi) \times P(S \mid \phi)$ & & 0.3302 & $0.3302 /(0.3302+0.3674)=\mathbf{0 . 4 7 3}(1)$ \\
\hline$P($ IBD at locus $A$ given marker identity) & & from 10,000 simulations: $\mathbf{0 . 4 6 8}$ \\
\hline
\end{tabular}

(a) First (last) position denotes IBD status of locus $A$ (marker), and $\mathrm{x}$ or _ denotes recombination or no recombination, respectively, between the loci.

Because of symmetry, $P(\phi=[0 \times 1])=P(\phi=[1 \times 0])$. The last IBD vector that we need to consider is $\phi=[0 \mathrm{x} 0]$. The probability of the first locus being nonIBD is $(1-\mathrm{f}(0))$. Next we need the probability that the second locus is nonIBD $(\phi(3)=0)$ given that the first locus is nonIBD:

$$
\begin{aligned}
P(\phi(3)=0 \mid \phi(1)=0) & =1-P(\phi(3)=1 \mid \phi(1)=0) \\
& =1-P(\phi(3)=1 \& \phi(1)=0) /(1-\mathrm{f}(0)) \\
& =1-\mathrm{f}_{\mathrm{r}}(0, c),
\end{aligned}
$$

where the latter identity is from equation (6). Combining these probabilities yields,

$$
P(\phi=[0 \mathrm{x} 0])=(1-\mathrm{f}(0))\left(1-\mathrm{f}_{\mathrm{r}}(0, c)\right)
$$

All $P(\phi)$ are calculated from equations (4-8) to get the probability of locus $A$ and AIS indicator $S$, i.e., $P(S \&$ locus $A)$, from equation (2). The $P(S \&$ locus $A)$ with IBD and nonIBD locus $A$ are combined in equation (1) to obtain the probability that locus $A$ is IBD given the linked marker haplotype. An example of the calculation of the IBD probability at locus $A$ is given in Table II.

\subsection{IBD probability at locus $\boldsymbol{A}$ given multiple linked markers}

Here we consider the situation where locus $A$ is surrounded by a marker haplotype, i.e., there are several linked markers. With several markers, equation (1) remains the same, except that the marker information is now due to 
several markers. Hence, $S$ is now a $(m \times 1)$ vector of AIS status indicators, where $m=$ the number of marker loci in the haplotype. The order of the elements in $S$ is assumed the same as the order of the loci on the chromosome. Also the $\phi$ vector is extended by adding two characters for every additional locus, one indicating whether the region between this locus and the previous locus was inherited en bloc from a common ancestor, "_," or not, "x", and one character indicating whether the locus is IBD, " 1 ", or nonIBD, " 0 ". Having more marker loci does not change equation (2), except that the number of possible $\phi$ vectors is substantially increased. Given IBD statuses at the loci, the probabilities of the elements of $S$ are independent, i.e.,

$$
P(S \mid \phi)=\prod_{\text {marker loci } i} P(S(i) \mid \phi(\text { at locus } i)) .
$$

Less straightforward is the evaluation of the probability of this larger vector of IBD statuses, $P(\phi)$. Let us first study the straightforward application of the method of the previous section to the example with $\phi=\left[1 \_1 \times 1\right]$, equidistant loci of $0.01 \mathrm{M}$ apart and the first locus being locus $A$. This $\phi$ vector contains an IBD region of $0.01 \mathrm{M}$, followed by a recombination in a region of $0.01 \mathrm{M}$, with probability $\mathrm{f}_{\mathrm{r}}(0.01,0.01)$. Next follows an IBD locus, which is assumed independent due to the recombination with probability $\mathrm{f}(0)$. Hence, the total probability is $\mathrm{f}_{\mathrm{r}}(0.01,0.01) \times \mathrm{f}(0)$. However, if we evaluate this $\phi$ vector from right to left, we would first have a region of size 0 followed by a recombination, with probability $\mathrm{f}_{\mathrm{r}}(0,0.01)$, which is followed by an IBD region of size 0.01 , yielding a total probability of $\mathrm{f}_{\mathrm{r}}(0,0.01) \times \mathrm{f}(0.01)$. These two probabilities are only approximately the same. The probabilities differ because of the assumption of independence after a recombination has occurred, which is only approximately true (see 4. DISCUSSION). Note that the first evaluation of $P(\phi)$ accounts for the recombination which ends the IBD region of locus $A$ (the first locus here), whereas the second evaluation of $P(\phi)$ attributes this recombination to the IBD region that surrounds the third locus. Because we are primarily interested in the IBD probability of locus $A$, it is important to accurately account for the size of the IBD region that contains locus $A$, i.e. the locus $A$ region. Hence, we account for the recombinations that end the locus $A$ region (if any) while evaluating $P(\phi)$.

The above is achieved by evaluating the locus $A$ region first and accounting for any recombination that ends this region. Next, we evaluate the remaining haplotype to the right of locus $A$, which is evaluated from left to right. Lastly, we evaluate the remaining haplotype to the left of locus $A$, which is evaluated from right to left. The rules for evaluating $P(\phi)$ are: 
1. If locus $A$ is nonIBD, set $P(\phi)=1-\mathrm{f}(0)$; otherwise if locus $A$ is on an IBD region of size $c$

- which ends due to recombinations on one side in a region of size $c_{1}$ and on the other side in a region of size $c_{2}$ : set $P(\phi)=\mathrm{f}_{\mathrm{dr}}\left(c, c_{1}, c_{2}\right)$;

- which ends on one side due to a recombination in a region of size $c_{1}$ : set $P(\phi)=\mathrm{f}_{\mathrm{r}}\left(c, c_{1}\right)$;

- which extends over the whole haplotype: set $P(\phi)=\mathrm{f}(c)$.

2. Evaluate the remaining haplotype to the right of the locus $A$ region from left to right. If the next characters of $\phi$ are:

- "x0", i.e. the next locus is nonIBD. If the last evaluated region was nonIBD: set $P(\phi)=P(\phi) \times\left(1-\mathrm{f}_{\mathrm{r}}(0, c)\right)$, where $c$ is the distance of the region corresponding to the $\mathrm{x}$ in " $\mathrm{x} 0$ "; otherwise if the last evaluated region was IBD: set $P(\phi)=P(\phi) \times(1-\mathrm{f}(0))$, i.e. the recombination was already accounted for when evaluating this IBD region;

- "x1(_1) $)^{n} \mathrm{x}$ " where (_1) ${ }^{n}$ denotes $n$ repetitions of the "_1" string $(n=$ $0,1,2, \ldots)$, i.e. the next region is an IBD region of size $c$, which is delimited by two recombinations. If the last evaluated region was nonIBD, account for both recombinations and set: $P(\phi)=P(\phi) \times \mathrm{f}_{\mathrm{dr}}\left(c, c_{1}, c_{2}\right)$, where $c_{1}\left(c_{2}\right)=$ the size of the region corresponding to the first (last) " $\mathrm{x}$ " in the string " $\mathrm{x} 1\left(\_1\right)^{n} \mathrm{x}$ ". Otherwise if the last evaluated region was IBD, the first recombination was already accounted for when evaluating this previous IBD region and set

$$
P(\phi)=P(\phi) \times \mathrm{f}_{\mathrm{r}}\left(c, c_{2}\right) ;
$$

- "x1(_1 $)^{n}$ ", i.e. the haplotypes end with an IBD region of size $c$. If the previously evaluated region was nonIBD, we should account for the recombination and set $P(\phi)=P(\phi) \times \mathrm{f}_{\mathrm{r}}\left(c, c_{1}\right)$, where $c_{1}$ is the size of the region in which the recombination occurred. If the previously evaluated region was IBD, we set $P(\phi)=P(\phi) \times \mathrm{f}(c)$.

The above types of regions (matching strings of $\phi$ ) are evaluated until the end of the haplotype ( $\phi$ ends).

3. Evaluate the haplotype that remains to the left of the locus $A$ region from right to left. This step is basically the mirror image of Step 2 and is not written out here to avoid repetition, but, for completeness, is written out in detail in Appendix A.

The above method will be illustrated by the example of Table III, where two markers surround locus $A$. The distance between the markers is $1 \mathrm{cM}$ and locus $A$ is in the middle between the markers. The gametes for which the IBD probability at locus $A$ is estimated carry identical marker alleles for both markers. The IBD status 1_1_1 (see Tab. III) denotes that the entire $1 \mathrm{cM}$ region is IBD, which equals $\mathrm{f}(0.01)=0.18221$ (equation (3)). The IBD status 
Table III. Calculation of IBD probability between two gametes at locus $A$ given that two linked markers that bracket locus $A$ have identical alleles.

The distance between the markers is $0.01 \mathrm{M}$ and locus $A$ is in the middle of this bracket. The effective size and time since the base population were both 100 , and initial homozygosity of the markers was 0.5 . Equation numbers are in parentheses ().

\begin{tabular}{|c|c|c|c|}
\hline IBD-status $(\phi)^{\text {(a) }}$ & $P(\phi)$ & $\begin{array}{l}A \text { is IBD: } \\
P(S \mid \phi)(9)\end{array}$ & $\begin{array}{c}A \text { is nonIBD: } \\
P(S \mid \phi)(9)\end{array}$ \\
\hline 1_1_1 & 0.18221 & 1 & - \\
\hline 1_1x1 & 0.03002 & 1 & - \\
\hline 1_1x0 & 0.04616 & 0.5 & - \\
\hline 1x1_1 & 0.03002 & 1 & - \\
\hline $1 \times 1 \times 1$ & 0.00934 & 1 & - \\
\hline $1 \times 1 \times 0$ & 0.01437 & 0.5 & - \\
\hline $1 x 0 x 1$ & 0.01124 & - & 1 \\
\hline $1 \mathrm{x} 0 \mathrm{x} 0$ & 0.07142 & - & 0.5 \\
\hline 0x1_1 & 0.04616 & 0.5 & - \\
\hline $0 \times 1 \times 1$ & 0.01437 & 0.5 & - \\
\hline $0 \times 1 \times 0$ & 0.02209 & 0.25 & - \\
\hline $0 x 0 x 1$ & 0.07142 & - & 0.5 \\
\hline $0 \times 0 \times 0$ & 0.45365 & - & 0.25 \\
\hline \multicolumn{2}{|c|}{$\sum P(\phi) \times P(S \mid \phi)$} & 0.31764 & 0.19607 \\
\hline \multicolumn{2}{|c|}{$P($ IBD at locus $A$ given marker identity): } & \multicolumn{2}{|c|}{$\begin{array}{l}0.31764 /(0.31764+0.19607) \\
=\mathbf{0 . 6 1 8}(1) \\
\text { from } 10,000 \text { simulations: } \mathbf{0 . 6 1 5}\end{array}$} \\
\hline
\end{tabular}

(a) Digits denote IBD status of left marker, locus $A$, and right marker, respectively. The $\mathrm{x}$ or _ denotes recombination or no recombination, respectively, between the loci.

1_1x1 denotes: i) an IBD region of $0.5 \mathrm{cM}$, with a recombination in the next $0.5 \mathrm{cM}$ region (probability is $\left.\mathrm{f}_{\mathrm{r}}(0.005,0.005)=0.0761\right)$; ii) an IBD locus at the second marker (probability is $\mathrm{f}(0)=0.394$ ), i.e. the total probability of IBD status $1 \_1 \times 1$ is $0.0761 \times 0.394=0.03002$. Because of symmetry this also equals the probability of the IBD status $1 \times 1 \_1$. The calculation of the IBD status 1_1 10 is similar, except that here the second marker locus is nonIBD (probability is $(1-\mathrm{f}(0))=0.606)$, and the total probability is thus $0.606 \times 0.0761=0.04616$.

The IBD status $1 \times 1 \times 1$ of Table III is IBD at the locus $A$ region which is $0 \mathrm{M}$, and has a recombination to the left and right in a region of size $0.5 \mathrm{cM}$ (probability is $\left.\mathrm{f}_{\mathrm{dr}}(0,0.005,0.005)=0.06\right)$. To the right, we still have to account for the IBD region of size 0 at the rightmost marker locus (probability is $\mathrm{f}(0)=0.394)$. Similarly to the left we still have to account for an IBD region 
of size 0 at the leftmost locus $(\mathrm{f}(0))$. Hence, the total probability of $1 \times 1 \times 1$ is $0.06 \times 0.394^{2}=0.00934$. Similarly, the IBD status $1 \times 1 \times 0$ has probability $\mathrm{f}_{\mathrm{dr}}(0,0.005,0.005)(1-\mathrm{f}(0)) \mathrm{f}(0)=0.1437$. And the probability of $0 \mathrm{x} 1 \mathrm{x} 0$ is $\mathrm{f}_{\mathrm{dr}}(0,0.005,0.005)(1-\mathrm{f}(0))^{2}=0.02209$.

Next, we consider the IBD status $1 \times 0 x 1$. We start with evaluating the locus $A$ region, which is non-IBD, with probability $(1-\mathrm{f}(0))=0.606$. To the right of locus $A$ there is a recombination in a region of $0.5 \mathrm{cM}$ and next an IBD marker locus with probability $\mathrm{f}_{\mathrm{r}}(0,0.005)=0.136$. An identical IBD status is found to the left of locus $A$. Hence, the total probability of $1 \times 0 \times 1$ is $0.606 \times 0.136^{2}=$ 0.1124 . If we consider the IBD status $1 \times 0 \times 0$, there is a nonIBD marker locus to the right of locus $A$ (probability is $\left(1-\mathrm{f}_{\mathrm{r}}(0,0.005)\right.$; equation (7)). Hence, the probability of $1 \mathrm{x} 0 \mathrm{x} 0$ is $0.606 \times(1-0.136) \times 0.136=0.07142$. Similarly, $0 \times 0 \times 0$ has probability $0.606 \times(1-0.136)^{2}=0.45365$. Because of symmetry, the probabilities of $0 \times 1 \_1,0 \times 1 \times 1$, and $0 x 0 x 1$ equal those of $1 \_1 \times 0,1 \times 1 \times 0$, $1 \mathrm{x} 0 \mathrm{x} 0$, respectively.

In the above, all the $P(\phi)$ terms of Table III were calculated and Table III shows that they resulted in an IBD probability at locus $A$ of 0.618 , which is close to the simulated value of 0.615 (the simulation is explained in Sect. 2.4 Testing the prediction of IBD probabilities). Appendix B gives an algorithm to calculate $P$ (nonIBD \& markers), where as many as possible terms are factored out in the summations of equations (2). The latter is important because the number of terms in summation (2) increases exponentially with the number of markers, and the calculation would become slow when the number of linked markers exceeds about 15 .

\subsection{Including pedigree information}

Generally the information on markers splits the pedigree into two parts:

1. generations where neither pedigree nor marker data is available (current marker data can be used to predict IBD probabilities due to these generations, as shown in the previous sections). This pedigree part results in linkage disequilibria between marker haplotypes and locus $A$ in the first generation of the pedigreed population and thus contains the LD information;

2. generations with known pedigree and marker data, although the marker information may be missing on some individuals. Wang et al. [23] presented a method that approximates the IBD probabilities given pedigree and marker information where the marker data may be incomplete (for recent developments and review see [1]). Exact IBD probabilities may be obtained by segregation analysis [3] or estimated by Gibbs sampling [5] (for recent developments and review [16]), but these methods are computationally very demanding when the number of loci is large and the pedigree is large and contains many loops. This pedigree part contains the linkage information, 
the inheritance of the markers and locus $A$ are traced through the known pedigree and the frequency with which recombinations occur yields information about the linkage between locus $A$ and the markers.

In practice, pedigree recording often started earlier than genotyping such that pedigree part 2 will often consist of some generations of pedigree recorded but non-genotyped individuals followed by generations of genotyped and pedigree recorded individuals. The approximation of Wang et al. [23] will become computationally demanding because it involves summation over many unknown genotypes in situations where none of the close relatives are genotyped. Also this approximation only uses the markers that flank locus $A$ to infer IBD probabilities, which ignores information in situations where the haplotypes consist of many closely linked markers and are sufficiently informative to infer whether there was a common ancestor or not. Here we developed another approximation to calculate IBD probabilities given marker and pedigree information, in the situation where the pedigree of the genotyped animals is known for some generations, but the individuals in this pedigree are not genotyped. The method presented will make better use of the information contained in the marker haplotype than Wang et al., but it will only consider the two haplotypes for which the IBD probability is required while Wang et al. considered all marker genotyped animals simultaneously. The latter will mainly be an advantage when for instance some non-genotyped sires have many genotyped offspring such that the genotypes of the sires can be inferred from their genotyped offspring.

We used an approach analogous to Wright's [25] $F$-statistics here, where marker haplotypes are related due to a finite population size for $T$ generations (pedigree part 1, Wright's $F_{\mathrm{ST}}$ ), and some marker haplotypes are related due to relationships in the pedigree (pedigree part 2; Wright's $F_{\text {IS }}$ ). The total IBD probability of locus $A$ given the one generation of marker haplotypes and some ancestral generations of pedigree is (analogous to Wright's $F_{\mathrm{IT}}$ ) :

$P_{\mathrm{IT}}(\mathrm{IBD} \mid$ marker, pedigree $)=P_{\mathrm{IS}}(\mathrm{IBD} \mid$ marker, pedigree $)$

$$
+\left[1-P_{\mathrm{IS}}(\mathrm{IBD} \mid \text { marker, pedigree })\right] P(\mathrm{IBD} \mid \text { marker }),
$$

where $P_{\mathrm{IS}}(\mathrm{IBD} \mid$ marker, pedigree $)=$ the IBD probability at locus $A$ due to a common ancestor within the pedigree and given the marker information (i.e., due to recent relationships); and $P(\mathrm{IBD} \mid$ marker $)=$ the probability that two regions are IBD before they entered the pedigree, i.e., due to $T$ generations of random drift in a population of size $N_{\mathrm{e}} . P(\mathrm{IBD} \mid$ marker) is obtained from equation (1) as described above. $P_{\mathrm{IS}}$ (IBD|marker, pedigree) can also be obtained from equation (1), but with equation (3) replaced by $f_{\mathrm{IS}}(c)$, where $f_{\mathrm{IS}}(c)$ is the probability that a region of size $c$ is IBD within the pedigree without the use of marker information $\left(\right.$ e.g. $f_{\mathrm{IS}}(0)$ is a coancestry coefficient given the 
pedigree information). Several algorithms are available that calculate $f_{\text {IS }}(c)$ in a pedigree $[7,18,22]$. This method of predicting $P_{\mathrm{IS}}(\mathrm{IBD} \mid$ marker, pedigree) uses only the two haplotypes for which the IBD probability is to be calculated to predict the haplotypes of the common ancestors in the pedigree, which may be little information if the haplotypes are not very informative (few not very informative markers).

\subsection{Testing the prediction of IBD probabilities at locus $A$}

The prediction of IBD probabilities given the information from markers was tested by the genedropping method [11]. In the genedropping method, the inheritance of linked marker alleles and founder alleles at locus $A$ is simulated in a pedigree, i.e., every offspring obtains at random one of the alleles of its sire and its dam, and with probability $(1-r)$ the linked allele at the next locus or with probability $r$ the alternative allele that is not in linkage phase, where $r$ is the recombination rate between the loci which is based on the Haldane [8] mapping function. The pedigree is obtained by randomly sampling for each of $N_{\mathrm{e}}$ offspring a sire and a dam, starting at the second generation $(T-1$ generations ago) until the current generation. For locus $A$, founder alleles are assumed in the base generation ( $T$ generations ago), i.e. all $2 N_{\mathrm{e}}$ alleles are different. If two alleles are identical at locus $A$ in later generations, they are a copy of the same founder allele and thus IBD. For the marker loci, the allele frequencies of the base population are assumed known, and marker alleles are sampled from this distribution of alleles, which assumes Hardy-Weinberg genotype frequencies.

Consider the locus order $[A, X, Y]$, where $X$ and $Y$ are marker loci, and consider that the marker alleles at locus $X$ are non-identical for two haplotypes, which implies that locus $X$ is nonIBD. The latter also implies that a possible IBD region around locus $A$ must end before locus $X$, and that the IBD status of locus $A$ is independent of that of locus $Y$. Hence, if the marker alleles at locus $X$ are non-identical, the identity status of locus $Y$ does not affect the IBD probability of locus $A$. This suggests a grouping of the IBD probabilities of haplotypes, namely all haplotype pairs that have a continuous string of $a$ identical marker alleles to the left of locus $A$ and a continuous string of $b$ identical marker alleles to the right of locus $A$ have the same IBD probability. For example, the haplotype pair $(1,1,1,1, A, 1,1,1,1)$ and $(2,2,1,1$, $A, 1,1,2,2)$ have the same IBD probability at locus $A$ as the pair $(2,2,2$, $2, A, 2,2,2,2)$ and $(2,3,2,2, A, 2,2,3,2)$ (assuming unknown initial allele frequencies), since both pairs have $(a, b)$ equal to $(2,2)$. Because of this grouping of haplotype pairs into groups that have equal IBD probabilities, we can compare estimated and predicted IBD probabilities for these groups instead of for individual haplotypes. 
The estimation of the IBD probability at locus $A$ of haplotype pairs from the genedropping is:

$$
\begin{array}{r}
P(\text { locus } A=\mathrm{IBD} \mid \text { haplotype }(a, b)) \\
=\frac{\sum_{i} \sum_{j} \sum_{k \neq j} I\left[\left(H_{i j} ; H_{i k}\right)=(a, b) \& \text { locus } A=\mathrm{IBD}\right]}{\sum_{i} \sum_{j} \sum_{k \neq j} I\left[\left(H_{i j} ; H_{i k}\right)=(a, b)\right]}
\end{array}
$$

where $\sum_{i}$ denotes summation over replicated simulations; $\sum_{j}\left(\sum_{k \neq j}\right)$ denotes summation over the haplotypes of the animals after $T$ generations of simulation; $I\left[\left(H_{i j} ; H_{i k}\right)=(a, b)\right]$ is an indicator variable which is one if the haplotype pair $H_{i j}$ and $H_{i k}$ belong to group $(a, b)$ and 0 otherwise; $I\left[\left(H_{i j} ; H_{i k}\right)=(a, b) \& \operatorname{locus} A=\mathrm{IBD}\right]$ is an indicator variable which is 1 if $H_{i j}$ and $H_{i k}$ belong to group $(a, b)$ and the founder alleles at locus $A$ are identical, i.e., locus $A$ is IBD, and 0 otherwise.

\section{RESULTS}

\subsection{No pedigree information}

Table IV shows predicted and simulated IBD probabilities of haplotype pairs that have $a(b)$ identical markers to the left (right) of the QTL, in the case of founder alleles at the markers, i.e. in the base population all marker alleles were different from each other and probability of alike in state, $a_{i}=0$. The haplotype consisted of 10 equidistant markers that were $1 \mathrm{cM}$ apart, and locus $A$ was in the middle of this marker haplotype, i.e. in the middle between the 5 th and 6th marker. Due to the symmetry of the haplotypes, the IBD probabilities are equal for haplotype pairs belonging to group $(a, b)$ and $(b, a)$. If none of the markers are identical, i.e., haplotype group $(0,0)$, locus $A$ can only be IBD due to a double recombination between its adjacent markers, which happens with a low probability of $4.7 \%$. If some markers are identical to, say, the left and none to the right of locus $A$, i.e. group $(a, 0)$ with $a>0$, some recombination must have occurred between the markers that are adjacent to locus $A$. If only one recombination occurred between these adjacent markers, this recombination occurred with a probability of $50 \%$ to the right of locus $A$, yielding an IBD locus $A$, or to the left of the locus, yielding a nonIBD locus $A$. Due to the probability of a double recombination, the IBD probability of locus $A$ is somewhat smaller than 0.5 for the haplotype group $(a, 0)$. If there are some founder marker alleles identical to the left and to the right of locus $A$, i.e., group $(a, b)$ with $a>0$ and $b>0$, there is an IBD region to the left and to the right of locus $A$, and locus $A$ can only be nonIBD by a double recombination. 
The deviations of the IBD probabilities of these haplotype groups from 1 are thus due to the double recombination probability. This suggests that the IBD probabilities would be identical for all $(a, b)$ groups with $a>0$ and $b>0$, since the double recombination probabilities are identical. The latter is however not the case, because a large IBD region, i.e., many markers equal to the left and right, is probably due to a recent common ancestor of the haplotype, which reduces the number of meiosis during which the two recombinations could have occurred. Hence, the IBD probabilities increase with the number of identical markers to the left and to the right.

The accuracy of the predictions of IBD probabilities seems reasonable, with deviations from the simulated probabilities ranging from -0.028 to 0.023 (Tab. IV). Some trend can be observed namely that IBD probabilities of $(a, b)$ haplotype groups with $a>0$ or $b>0$ and small $a$ and $b$ are somewhat overpredicted, i.e. genedropping minus predicted probabilities are negative.

The situation that is shown in Table V is very similar to that in Table IV, except that the marker loci are bi-allelic with equal expected allele frequencies in the base population, i.e. markers have an alike in state probability of $a_{i}=0.5$. The reduced information content of the markers decreased the IBD probabilities at locus $A$, because it is now possible for markers to be identical in state but not identical by descent. The deviations of the predicted and genedropping IBD probabilities at locus $A$ ranged from -0.016 to +0.018 , and are somewhat smaller than in Table IV. There is a tendency for haplotype groups with $a>0$, $b>0$ and small $a$ and $b$ to have underpredicted IBD probabilities, which is opposite to the trend in Table IV. Since the sign of the deviations is often opposite between Tables IV and V, it may be expected that the deviations will be smaller for intermediate $a_{i}$ values, i.e. $0<a_{i}<0.5$, which would hold for most micro-satellite markers.

Table VI shows accuracies of prediction of the IBD probabilities at locus $A$ for inter-marker distances ranging from $0.25-40 \mathrm{cM}$. Although 10 markers spaced at $40 \mathrm{cM}$ intervals is not realistic it was thought desirable to test the accuracy of prediction in extreme cases. The accuracies are expressed as square roots of the mean square error of prediction $(\sqrt{M S E P})$. In general, the accuracies of the predictions are similar to those at an inter-marker distance of $1 \mathrm{cM}$. However, in the case of fully informative markers and large inter-marker distances of 20 and $40 \mathrm{cM}$, the accuracy of prediction of IBD probabilities is substantially reduced. This reduced accuracy is mainly because the IBD probabilities of haplotype groups $(a, b)$ with large $a$ and $b$ are substantially underpredicted (result not shown). With these large inter-marker distances, the probability of a double recombination within a bracket and meiosis is substantial. The latter implies that after a first recombination, a second recombination can occur which reverses the effect of the first recombination. Hence, the probability of no recombination, $\exp (-c)$, in the derivation of 


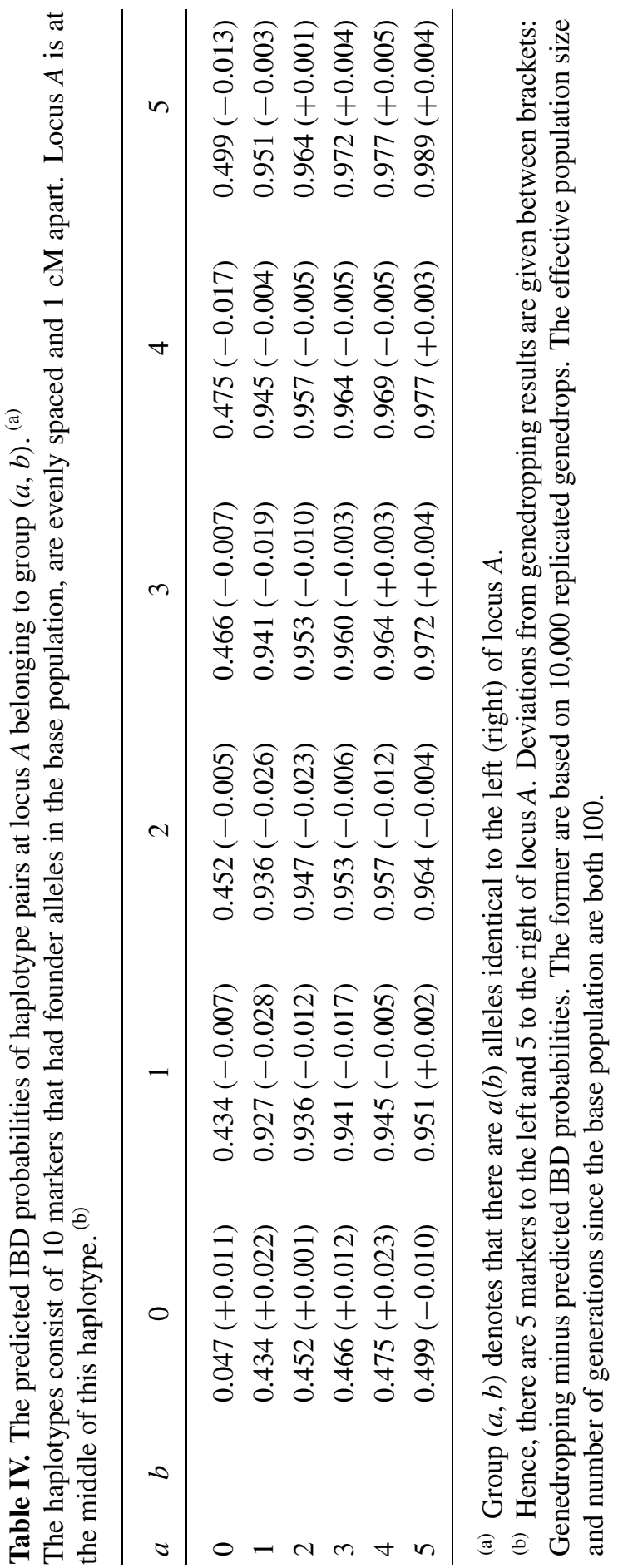




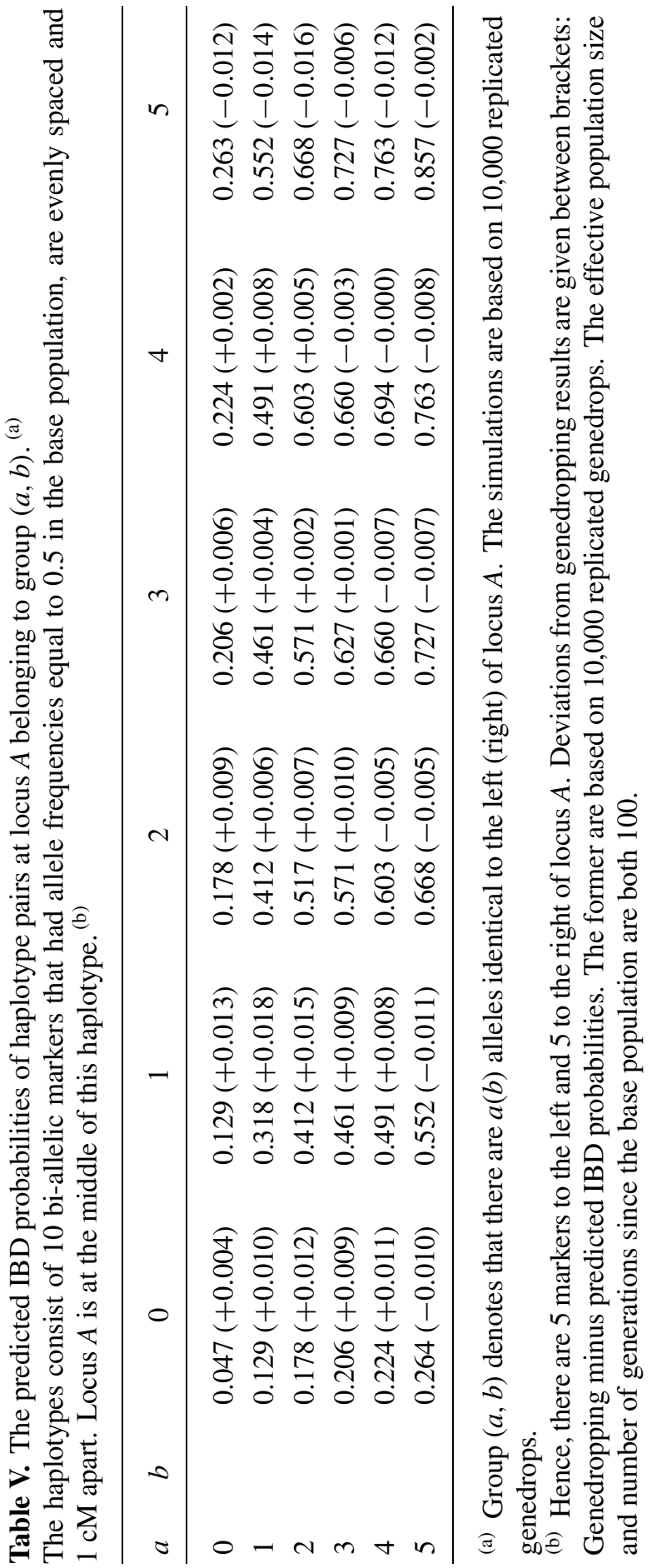


Table VI. Square root of the mean square error of prediction of IBD probabilities $(\sqrt{M S E P})$ at locus $A .^{\text {(a) }}$

The haplotypes consist of 10 equidistant markers. Locus $A$ is at the middle of this haplotype.

\begin{tabular}{lcc}
\hline $\begin{array}{l}\text { Between marker } \\
\text { distance }\end{array}$ & $\begin{array}{c}\text { Markers with } \\
\text { founder alleles }\end{array}$ & $\begin{array}{c}\text { Bi-allelic markers } \\
\text { (initial frequency }=0.5)\end{array}$ \\
\hline $0.25 \mathrm{cM}$ & 0.009 & 0.014 \\
0.5 & 0.008 & 0.011 \\
1 & 0.012 & 0.009 \\
5 & 0.019 & 0.010 \\
10 & 0.022 & 0.008 \\
20 & 0.034 & 0.008 \\
40 & 0.070 & 0.005 \\
\hline
\end{tabular}

(a) $\sqrt{M S E P}=\sqrt{\left[\sum\left(P\left(\mathrm{IBD}_{A}\right)_{\text {pred }}-P\left(\mathrm{IBD}_{A}\right)_{\text {sim }}\right)^{2} / 36\right]}$, where $P\left(\mathrm{IBD}_{A}\right)_{\text {pred }}$ and $P\left(\mathrm{IBD}_{A}\right)_{\text {sim }}$ are the IBD probabilities at locus $A$ obtained from prediction and 10,000 genedropping simulations, respectively; and summation is over all 36 haplotype groups $(a=0,1, \ldots, 5 ; b=0,1, \ldots, 5)$. The effective population size and number of generations since the base population are both 100 .

equation (3) should be replaced by the probability of having no recombination at the marker loci in the region that is evaluated. This would make equation (3) more complex. Furthermore, Table VI shows that the predictions of the IBD probabilities are quite good when the markers have $a_{i}=0.5$. The latter is because a map of sparse markers with substantial alike in state probabilities contains little information about the IBD probabilities at locus $A$, i.e. the predicted and simulated IBD probabilities are quite close to the inbreeding level of the population, $\mathrm{f}(0)=1-\exp \left(-T /\left(2 N_{\mathrm{e}}\right)\right)$. For instance, with biallelic markers, an inter-marker distance of $40 \mathrm{cM}$, and all marker alleles equal within the haplotype, the IBD probability at locus $A$ is only 0.424 , while the inbreeding level is 0.394 .

\subsection{Larger effective population size}

Table VII investigates the effect of a larger effective population size, $N_{\mathrm{e}}$. When $N_{\mathrm{e}}=1000$, the IBD probabilities were generally smaller than with $N_{\mathrm{e}}=100$, probably due to the reduced inbreeding levels. However, in the case of founder alleles and some equal marker alleles to the left and to the right of locus $A(a>0$ and $b>0)$, the IBD probabilities are increased and are close to 1 , which suggests that the probability of a double recombination between the equal markers is very small. This is probably because a double recombination that makes locus $A$ nonIBD between IBD marker positions requires that two 
Table VII. Predicted IBD probabilities of haplotype pairs at locus $A$ belonging to group $(a, b)$, when the effective population size is 1000 (a) $^{\text {(a) }}$

Results from 10 bi-allelic markers are after $\backslash$ and those from markers with founder alleles are before $\backslash$.

\begin{tabular}{ccccccc}
\hline$a b$ & 0 & 1 & 2 & 3 & 4 & 5 \\
\hline 0 & $0.008 \backslash 0.008$ & 0.020 & 0.028 & 0.033 & 0.037 & 0.051 \\
1 & 0.349 & $0.975 \backslash 0.052$ & 0.072 & 0.087 & 0.101 & 0.147 \\
2 & 0.372 & 0.980 & $0.986 \backslash 0.102$ & 0.126 & 0.148 & 0.226 \\
3 & 0.395 & 0.984 & 0.990 & $0.994 \backslash 0.158$ & 0.189 & 0.304 \\
4 & 0.415 & 0.986 & 0.993 & 0.996 & $0.997 \backslash 0.230$ & 0.384 \\
5 & 0.451 & 0.990 & 0.995 & 0.998 & 0.999 & $1.000 \backslash 0.720$ \\
\hline
\end{tabular}

(a) Group $(a, b)$ denotes that there are $a(b)$ alleles identical to the left (right) of locus $A$. The haplotype consists of 10 evenly spaced markers, $1 \mathrm{cM}$ apart, and locus $A$ is at the middle of the marker bracket.

haplotypes meet in one individual and recombine around locus $A$ where one haplotype is IBD to the left of locus $A$ and the other is IBD to the right of locus $A$ (assuming that the probability of a double recombination in one generation is negligible). The probability that these haplotypes are found in one individual is reduced when population size increases, and hence the probability of a double recombination reduces, which explains these increased IBD probabilities. These extreme IBD probabilities with high $N_{\mathrm{e}}$ and highly polymorphic markers seem ideal for gene or QTL mapping experiments.

\subsection{A simple half sib pedigree structure}

The genedropping was performed as before, with 100 generations of random selection and mating at an effective size of 100 (i.e. 50 males and 50 females), after which a 101th generation was simulated by mating each of the 50 sires to 2 randomly sampled dams (sampling with replacement), which resulted in two half-sib offspring per sire. Hence, the 101th generation consisted of 50 half sib families, containing 2 half sibs each. The paternally inherited haplotypes were compared to the other paternal haplotypes within the same half-sib family, and the haplotype pairs were assigned to $(a, b)$-haplotype groups as before. The IBD rate at locus $A$ within each group was compared to the predicted IBD probabilities in Table VIII.

Table VIII shows the predicted IBD probabilities at locus $A$ when the paternally inherited haplotypes of half sibs are compared, i.e. both haplotypes were inherited from the same sire which had two half sib offspring. In the absence of marker information, the IBD probability of locus $A$ at these 


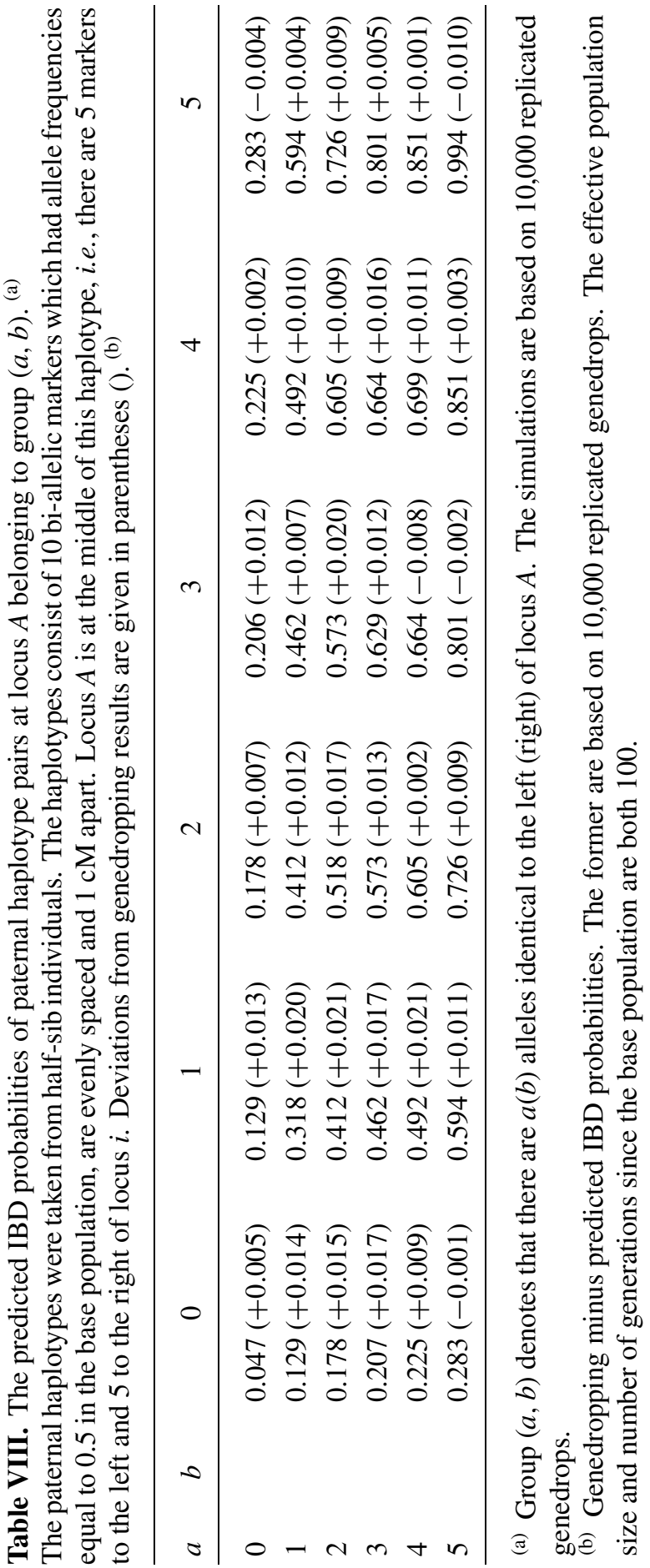


haplotypes is 0.5 . The probability that a region of $c$ Morgan is IBD is:

$$
f_{\mathrm{IS}}(c)=0.5 \exp (-2 c),
$$

where $\exp (-2 c)$ is the probability that no recombination occurred in either half sibs. The above formula for $f_{\mathrm{IS}}(c)$ replaces equation (3) to calculate $P_{\mathrm{IS}}\left(\mathrm{IBD} \mid\right.$ marker, pedigree), and the initial base generation homozygosity, $a_{0}$, is replaced by the homozygosity before entering the half sib pedigree, $a_{T}=$ $\mathrm{f}(0)+(1-\mathrm{f}(0)) a_{0}$, where $a_{T}$ and $\mathrm{f}(0)$ are the homozygosity and inbreeding, respectively, after 100 generations at an effective size of 100 .

The within half sib family IBD probabilities are very similar to the "unpedigreed" probabilities in Table V, except for $(a, b)$ haplotype groups with $a=5$ or $b=5$ or $a=b=5$. If $a=b=5$, i.e. the alleles were identical at all 10 marker loci, there seems to be sufficient evidence that both half sibs inherited the same haplotype from their sire and that this haplotype did not recombine since the IBD probability was very close to 1 . If, say, $a=5$ and $b<5$, the half sibs might still have inherited the same haplotype from their sire, but a recombination must have occurred since not all marker alleles were identical. This reduces the IBD probability at locus $A$ substantially especially when the non-identical marker alleles are close to locus $A$. However, the IBD probabilities are still larger than those in Table $\mathrm{V}$ for these haplotype groups. If there are non-identical marker alleles at both sides of locus $A$, i.e. $a<5$ and $b<5$, it is much less likely that the alleles at locus $A$ are a copy of the same locus $A$ allele of the sire since this would require a double recombination. Hence, if locus $A$ is still IBD, it will be IBD because the sire carried two alleles at locus $A$ which are IBD. This is as probable as the IBD probabilities in Table V. Hence, the IBD probabilities of Tables V and VIII are very similar when $a<5$ and $b<5$.

\section{DISCUSSION}

\subsection{Effects of multi-marker similarities on IBD probabilities}

The IBD probability at a predefined locus $A$ was predicted using the information from linked marker haplotypes and pedigree. The number of identical markers had a large effect on the IBD probability (see for example Tab. V), because a larger number of equal marker alleles: 1 ) decreases the probability of markers being identical by state; 2 ) indicates a more recent common ancestor and thus a smaller probability of double recombinations. The latter could render locus $A$ nonIBD even if the surrounding markers are IBD (Tab. IV). In the examples, we only considered haplotypes with equidistant markers and locus $A$ was in the middle of the haplotypes. The presented prediction method can, however, handle arbitrary distances between the loci, such that it can also 
predict IBD probabilities in more practical situations. To compare predicted versus simulated IBD probabilities when locus $A$ is not in the middle of the haplotype, we considered a locus $A$ between the 1st and 2nd marker of a marker haplotype as in Table V. This resulted in a $\sqrt{M S E P}$ of 0.008 (result not shown), which compares to the figure of 0.009 of Table VI for a mid-haplotype locus $A$, i.e. it seems that the accuracies of predicted IBD probabilities for loci that are or are not in the middle of their haplotypes is similar.

A complete simulation of the coalescence process [10,11] over multiple marker loci to estimate the IBD probabilities at locus $A$ would also account for the frequencies of the marker haplotypes. A very frequent haplotype indicates an old common ancestor and thus a considerable double recombination probability between locus $A$ and the markers. This information is not accounted for by the presented algorithm which considers only two haplotypes at a time.

Other factors affecting the IBD probability are shown in equation (3), which may be simplified to (assuming small $c$ and large $N_{\mathrm{e}}$ ):

$$
\mathrm{f}(c) \approx \frac{1-\exp \left[-T\left(\frac{1}{2 N_{\mathrm{e}}}+2 c\right)\right]}{1+4 N_{\mathrm{e}} c}
$$

which approaches $1 /\left(1+4 N_{\mathrm{e}} c\right)$ for large $T$. The latter equation equals the steady state LD between loci in a population of size $N_{\mathrm{e}}$ [20]. The above equation for $\mathrm{f}(c)$ shows that the IBD probabilities are expected to be equal in situations where $T\left(2 c+1 /\left(2 N_{\mathrm{e}}\right)\right)$ and $N_{\mathrm{e}} c$ are equal, i.e. where $N_{\mathrm{e}} c$ and $T / N_{\mathrm{e}}$ are equal. Hence, we expect that the comparisons between predicted and simulated IBD probabilities of Tables IV, $\mathrm{V}$ and VI will also hold for larger $T$, $N_{\mathrm{e}}$ or smaller $c$ as long as $N_{\mathrm{e}} c$ and $T / N_{\mathrm{e}}$ are equal to the values used in these tables.

The choice of the time since the base population, $T$, is arbitrary and similar to the situation where inbreeding coefficients are calculated from a known pedigree. As the assumed $T$ increases, the IBD probabilities increase. But simulation results show that LD mapping of QTL is very robust against the assumption about $T$ [14].

\subsection{Recombination makes next linked locus independent}

It was assumed that a recombination made the next locus independent from the previous IBD region, i.e. $P(Y=\mathrm{IBD} \mid X=\mathrm{IBD}$; recomb. $)=\mathrm{f}(0)$ where $X$ and $Y$ are two linked loci. Figure 1 shows genedropping results where $P(Y=\mathrm{IBD} \mid X=\mathrm{IBD}$; recomb. $)$ is plotted against the time at which the most recent recombination occurred, given that the common ancestor of locus $X$ lived 100 generations ago (the latter gives the largest differences of IBD probabilities over time). It appears that $P(Y=\operatorname{IBD} \mid X=\mathrm{IBD}$; recomb. $)>\mathrm{f}(0)=0.394$ 


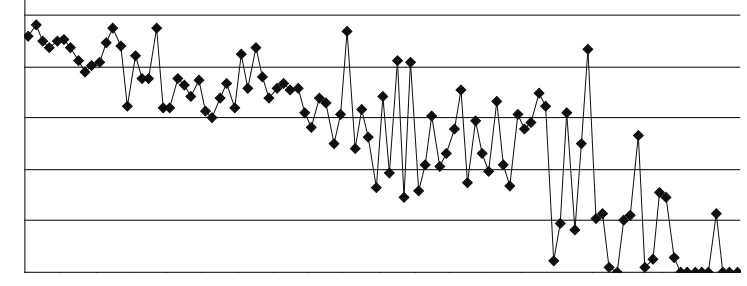

The recombination rate between locus $X$ and $Y$ was 0.01 . Results are based on 100,000 replicated genedrops. The erratic pattern in old generations is due to the infrequent occurrence of these situations.

Figure 1. The IBD probability at locus $Y$ given that a linked locus $X$ is IBD due to a common ancestor, which lived 100 generations ago, and given that a recombination occurred $G$ generations ago at the genetic path between the current haplotypes and the common ancestor.

The population is 100 generations old and its effective size is 100 , which yields an average IBD probability of 0.394 .

when the recombination occurred less than 15-20 generations ago; and that $P(Y=\mathrm{IBD} \mid X=\mathrm{IBD}$; recomb. $)<\mathrm{f}(0)=0.394$ when the most recent recombination occurred $>25$ generations ago. Hence, $P(Y=\operatorname{IBD} \mid X=$ IBD; recomb.) clearly varies with the time since the most recent recombination. This might be because, if the most recent recombination occurred a long time ago, the inbreeding levels at the time of the recombination were lower than $\mathrm{f}(0)$, which is the inbreeding level in the current generation. If the recombination occurred recently, the IBD probability is higher than $\mathrm{f}(0)$, which is probably because the haplotype of the old common ancestor of locus $X$ has a higher frequency than a randomly sampled haplotype in the current generation. Hence, the assumption $P(Y=\mathrm{IBD} \mid X=\mathrm{IBD}$; recomb. $)=\mathrm{f}(0)$ seems on average approximately right and Tables IV-VIII also suggest that this assumption gives reasonably accurate predictions. For more accurate predictions and an improved understanding of the relationships between similarity of marker haplotypes and IBD probabilities, further research to relax this assumption is needed.

\subsection{Accounting for allele frequencies instead of homozygosity, $a_{i}$}

The probability that the marker alleles are alike in state, $a_{i}$, was assumed equal to the homozygosity in the base population. However, if at marker 
locus $X$, allele 1 was much more rare than allele 2 in generation 0 , then two haplotypes that contain both allele 1 are more likely IBD than two haplotypes that contain allele 2 . The information about allele frequencies can be accounted for by setting $P\left(S_{i}=1 \mid\right.$ locus $i$ nonIBD $)=q_{i j}^{2}$ instead of $a_{i}$ in equation (9), where $q_{i j}=$ the frequency of allele $j$ at marker locus $i$ and the haplotypes were identical for alleles $j$. Similarly, we set $P\left(S_{i}=0 \mid\right.$ locus $i$ nonIBD $)=2 q_{i j} q_{i k}$, where the two haplotypes had marker alleles $j$ and $k$ at locus $i$, and $j \neq k$. In theory the allele frequencies $q_{i j}$ refer to base population frequencies, but in practice only allele frequencies of recent generation are known, which yield perhaps a sufficiently accurate approximation.

\subsection{Several generations of marker data}

In the "including pedigree information" section, we showed how to account for pedigree part 1 and the first generation with marker data of pedigree part 2 . In practice pedigree part 2 will often contain several generations of genotyped and pedigreed individuals for which also IBD probabilities are required. For the later generations of pedigree part 2, the recurrence relationships of Fernando and Grossman [4], Goddard [6] (in the case of marker brackets), and Wang et al. [23] (in the case of incomplete marker information) can be used. These recurrence relationships calculate the IBD probabilities between the offspring based on the IBD probabilities between the parents and the inheritance of the markers that flank locus $A$. Usually, these methods assume unrelated haplotypes in the first generation to which they are applied, but these first generations' relationships can also be set equal $P_{\mathrm{IT}}(\mathrm{IBD} \mid$ marker, pedigree) of equation (10), which accounts for the relationships due to pedigree part 1 and the non-genotyped generations of part 2. This combination of equation (10) for the IBD probabilities of the first genotyped generation of pedigree part 2, and the recurrence relationships of, e.g., Wang et al. [23] for the later generations yields IBD probabilities that account for the LD (pedigree part 1) and for the linkage between markers and locus $A$ (pedigree part 2). The use of these IBD probabilities in a QTL mapping analysis by variance components (for a review see [9]) results in a combined linkage-LD mapping analysis.

\subsection{Comparison to other methods}

Methods for linkage mapping of QTL fall into three categories, those using the full likelihood, non-parametric linkage analysis methods, and the variance component methods. The latter use the markers and pedigree to identify QTL alleles that are IBD and then estimate the variance between the QTL alleles. The method proposed here is a natural extension of this approach in which similarity of marker haplotypes are used to estimate the probability that QTL alleles are IBD due to a common ancestor before the known pedigree. 
Most other methods for estimating IBD probabilities from LD amongst marker haplotypes simply multiply the likelihoods of single marker LD together (e.g. [21]) which ignores the dependencies between the markers within a haplotype, and most are designed for specific pedigree structures such as affected sib pairs [2]. The method that is closest to that presented here is decay of haplotype sharing (DHS; [13]). This method and ours are similar in that they both use the haplotype data by modelling the length of the chromosome that is inherited by descendants of a common ancestor. However the methods differ in the situations for which they are intended. McPeek and Strahs consider an allele, presumably rare, that causes disease and assume that all or many sufferers of the disease carry the allele and a small chromosome segment from a common ancestor. The situation we envisage is more general: there are two or more alleles at a segregating QTL and one cannot define the genotype of an animal from its phenotype due to other genes and environmental factors affecting the trait. Chromosomes carrying the same QTL allele may have a recent or distant common ancestor. The marker density may be high or not. If it is not high, there may be no common haplotype shared by all alleles of one type. However chromosomes carrying this allele will fall into groups of related haplotypes that descend from a more recent common ancestor, and the resulting LD may still provide considerable power in a QTL mapping experiment.

The methods differ technically in that our method specifically models the probability that part(s) of two haplotypes are IBD even though the gene of interest is not IBD. McPeek and Strahs [13] estimate the frequencies of haplotypes from the non-affected population, which serve as a control population.

By using the presented IBD probabilities for QTL mapping by variance components, the presented method can easily incorporate polygenic background and environmental factors that might affect the phenotype.

\section{REFERENCES}

[1] Abdel-Azim G., Freeman A.E., A rapid method for computing the inverse of the genetic covariance matrix between relatives for a marked Quantitative Trait Locus, Genet. Sel. Evol. 33 (2001) 153-174.

[2] Almasy L., Williams J.T., Dyer T.D., Blangero J., Quantitative Trait Locus detection using combined linkage/disequilibrium analysis, Genet. Epidem. (1999) 17 (Suppl. 1) S31-S36.

[3] Elston R.C., Stewart J., A general model for the analysis of pedigree data, Human Hered. 21 (1971) 523-542.

[4] Fernando R.L., Grossman M., Marker-assisted selection using best linear unbiased prediction, Genet. Sel. Evol. 21 (1989) 246-477.

[5] Gilks W.R., Richardson S., Spiegelhalter D.J., Markov chain Monte Carlo in practice, Chapman and Hall, London, 1996. 
[6] Goddard M.E., A mixed model for analyses of data on multiple genetic markers, Theor. Appl. Genet. 83 (1992) 878-886.

[7] Guo S.W., Computation of multilocus prior probability of autozygosity fro complex inbred pedigrees, Genet. Epidemiol. 14 (1997) 1-15.

[8] Haldane J.B.S., The combination of linkage values, and the calculation of distances between loci of linked factors, J. Genet. 8 (1919) 299-309.

[9] Hoeschele I., Uimari P., Grignola F.E., Zhang Q., Gage K.M., Advances in statistical methods to map quantitative trait loci in outbred populations, Genetics 147 (1997) 1445-1457.

[10] Hudson R.R., The sampling distribution of linkage disequilibrium under an infinite alleles model without selection, Genetics 109 (1985) 611-631.

[11] Hudson R.R., The how and why of generating gene genealogies, in: Takahata N., Clarck A.G. (Eds.), Mechanics of Molecular Evolution, Sinauer, Sunderland, 1993, pp. 23-36.

[12] Maccluer J.W., Vandeberg J.L., Read B., Ryder O.A., Pedigree analysis by computer simulation, Zoo Biol. 5 (1986) 147-160.

[13] McPeek M.S., Strahs A., Assessment of linkage disequilibrium by the decay of haplotype sharing, with application to fine scale genetic mapping, Am. J. Hum. Genet. 65 (1999) 858-875.

[14] Meuwissen T.H.E., Goddard M.E., Fine mapping of quantitative trait loci using linkage disequilibria with closely linked marker loci, Genetics 155 (2000) 421-430.

[15] Patterson A.H., Molecular dissection of quantitative traits: progress and prospects, Genome Res. 5 (1995) 321-333.

[16] Perez-Enciso M., Varona L., Rothschild, M.F., Computation of identity by descent probabilities conditional on DNA markers via a Monte Carlo Markov Chain method, Genet. Sel. Evol. 32 (2000) 467-482.

[17] Rabinowitz D., A transmission disequilibrium test for quantitative trait loci, Hum. Hered. 47 (1997) 342-350.

[18] Schaffer A.A., Computing probabilities of homozygosity by descent, Genet. Epidemiol. 16 (1999) 135-149.

[19] Spielman R., McGinnis R., Ewens W., Transmission test for linkage disequilibrium: the insulin gene region and insulin-dependent diabetes mellitus (IDDM), Am. J. Hum. Genet. 52 (1993) 506-516.

[20] Sved J. A., Linkage disequilibrium and homozygosity of chromosome segments in finite populations, Theor. Pop. Biol. 2 (1971) 125-141.

[21] Terwilliger J.D., A powerful likelihood method for the analysis of linkage disequilibrium between trait and one or more polymorphic marker loci, Am. J. Hum. Genet. 56 (1995) 777-787.

[22] Thompson E.A., Monte Carlo estimation of multilocus autozygosity probabilities, in: Proc. 1994 Interface Conference, SAS ${ }^{\circledR}$, Cary, NC, 1994, pp. 498-506.

[23] Wang T., Fernando R.L., Van der Beek S., Grossman M., Van Arendonk J.A.M., Covariance between relatives for a marked quantitative trait locus, Genet. Sel. Evol. 27 (1995) 251-274.

[24] Weller J.I., Kashi Y., Soller M., Power of daughter and granddaughter designs for determining linkage between marker loci and quantitative trait loci in dairy cattle, J. Dairy Sci. 73 (1990) 2525-2537. 
[25] Wright S., Evolution and the Genetics of Populations. Vol. 2: The Theory of Gene Frequencies, University of Chicago, Chicago, 1969.

\section{APPENDIX A}

\section{From right to left evaluation of $\boldsymbol{P}(\phi)$}

When starting at the region of Locus $A$ and evaluating the haplotype from right to left, the next elements to the left of the current haplotype can be (note that during the first loop through these possibilities, the term "last evaluated region" denoted the locus $A$ region):

- "0x" and the last evaluated region was nonIBD: set $P(\phi)=P(\phi) \times(1-$ $\mathrm{f}_{\mathrm{r}}(0, c)$ ), where $c$ is the distance of the region corresponding to the $\mathrm{x}$; otherwise if the last evaluated region was IBD: set $P(\phi)=P(\phi) \times(1-\mathrm{f}(0))$, i.e. the recombination was already accounted for when evaluating this IBD region.

- "x $1\left(\_1\right)^{n} \mathrm{x}$ " where $\left(\_1\right)^{n}$ denotes $n$ repetitions of the "_l" string. If the last evaluated region was nonIBD, account for both recombinations and set: $P(\phi)=P(\phi) \times \mathrm{f}_{\mathrm{dr}}\left(c_{2}, c_{1}, c_{3}\right)$, where $c_{1}\left(c_{3}\right)=$ the size of the region corresponding to the first (last) " $\mathrm{x}$ " in the string " $\mathrm{x} 1\left({ }_{-} 1\right)^{n} \mathrm{x}$ ", and $c_{2}$ is the size of the IBD region corresponding to " $1\left(\_1\right)^{n}$ " in " $\mathrm{x} 1\left({ }_{-} 1\right)^{n} \mathrm{x}$ ". Otherwise if the last evaluated region was IBD, the rightmost recombination was already accounted for when evaluating this previous IBD region and set $P(\phi)=$ $P(\phi) \times \mathrm{f}_{\mathrm{r}}\left(c_{2}, c_{1}\right)$.

- " $\left(1_{-}\right)^{n} 1 \mathrm{x}$ ", i.e. the haplotypes end with an IBD region. If the previously evaluated region was nonIBD, we should account for the recombination and set $P(\phi)=P(\phi) \times \mathrm{f}_{\mathrm{r}}\left(c_{2}, c_{1}\right)$, where $c_{1}$ is the size of the region in which the recombination occurred and $c_{2}$ is the size of the IBD region. If the previously evaluated region was IBD, we set $P(\phi)=P(\phi) \times \mathrm{f}\left(c_{2}\right)$.

The above types of regions (matching strings of $\phi$ ) are evaluated from right to left until the haplotype ends (beginning of $\phi$ ).

\section{APPENDIX B}

\section{Algorithm for the calculation of IBD probability of a pair of haplotypes at locus $\boldsymbol{A}$ given the identities of the marker alleles}

The following Fortran90 module "prob_haplo_m" contains the function "prob_haplo", which calculates the $P$ (nonIBD \& marker) probabilities of equation (1). Hence, two calls of "prob_haplo" are needed to evaluate equation (1): One with locus $A$ is IBD while the second with locus $A$ is nonIBD. The input of "prob_haplo" is:

npos $=$ total number of positions considered (number of markers plus 1 (for $\operatorname{locus} A)$ ); 
$\operatorname{pos} A=$ position number of locus $A$;

pcond $(0: 1, i)=$ prob. of the observed identity at position $i$ given that position $i$ is nonIBD (terms of equation (3)). If the marker alleles are identical $\operatorname{pcond}(1, i)=1$ and $\operatorname{pcond}(0, i)=h_{i}$, where $h_{i}$ is the initial homozygosity at locus $i$; if they are not identical $\operatorname{pcond}(1, i)=0$ and $\operatorname{pcond}(0, i)=1-h_{i}$. The IBD status at locus $A$ is assumed known when calculating $P$ (nonIBD \& marker) and, hence, $h_{\text {posA }}=0$;

$T=$ number of generations since the base population;

$N_{\mathrm{e}}=$ effective population size.

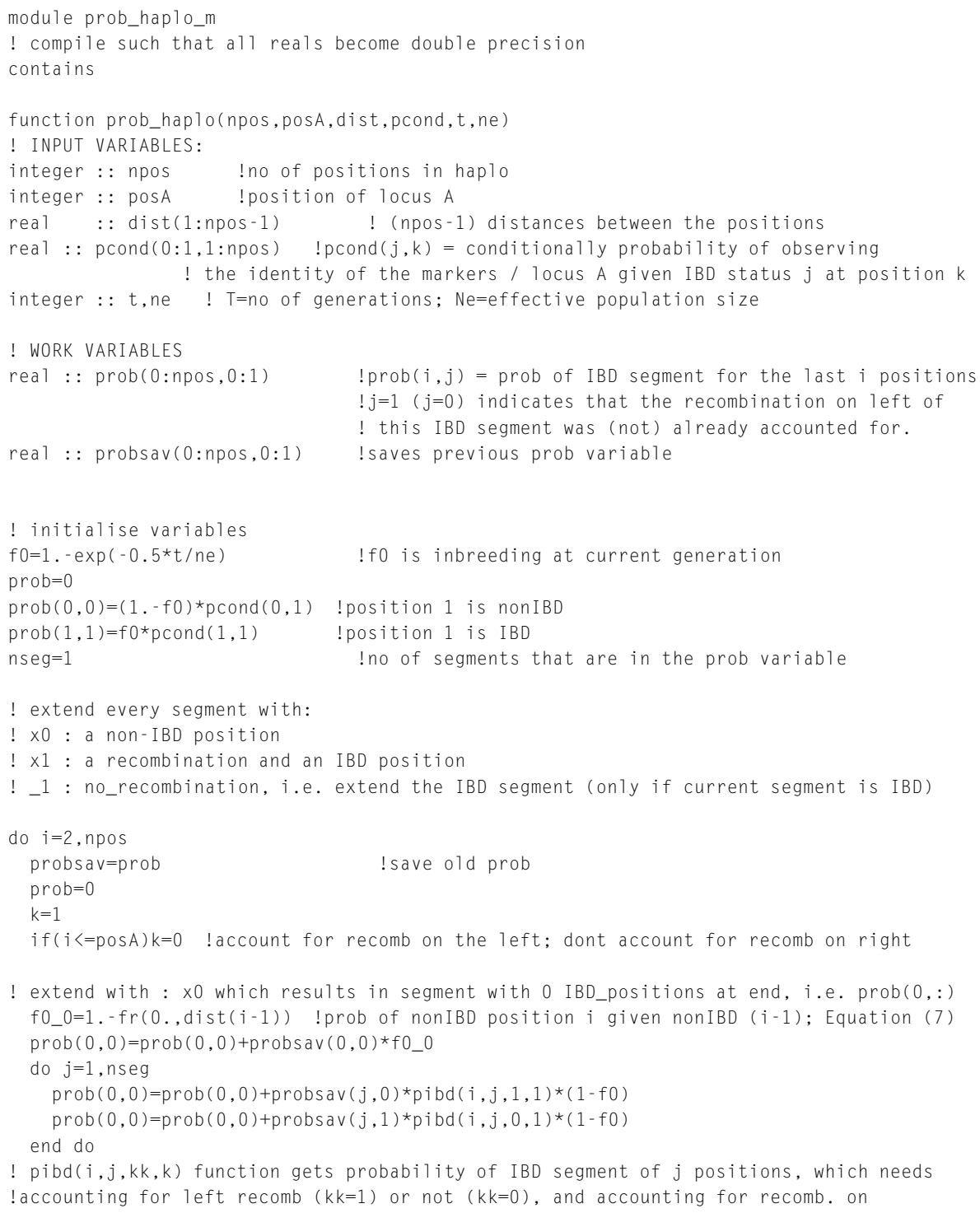




\section{IBD probabilities between marker haplotypes}

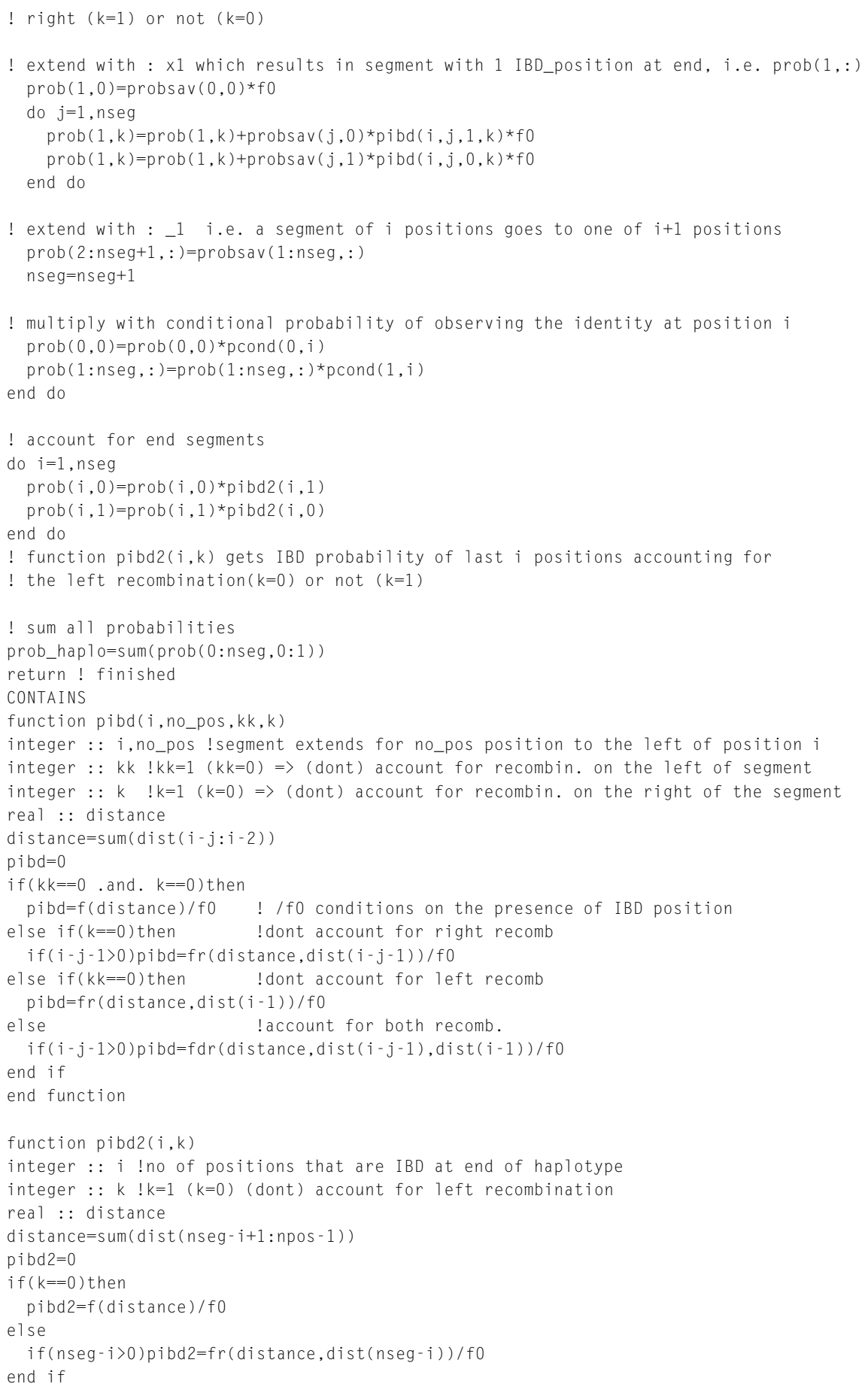




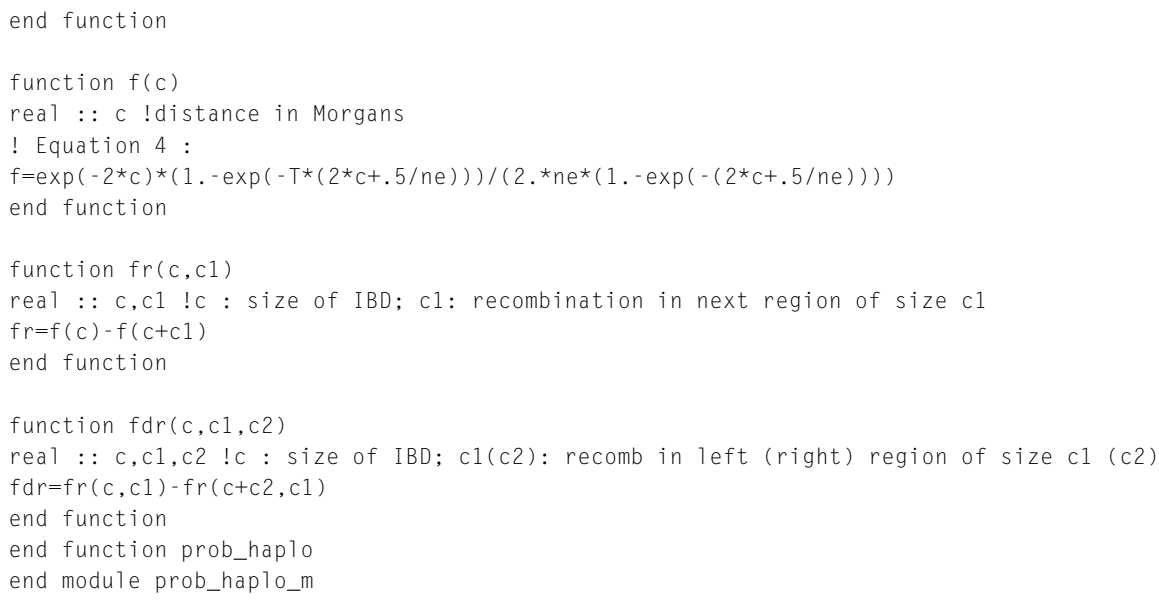

To access this journal online: www.edpsciences.org 